\title{
GENERATING GROUPS BY CONJUGATION-INVARIANT SETS
}

\author{
VALERY BARDAKOV, VLADIMIR TOLSTYKH, VLADIMIR VERSHININ
}

\begin{abstract}
Let $S$ be a generating set of a group $G$. We say that $G$ has finite width relative to $S$ if $G=\left(S \cup S^{-1}\right)^{k}$ for a suitable natural number $k$. We say that a group $G$ is a group of finite $C$-width if $G$ has finite width with respect to all conjugation-invariant generating sets of $G$. We give a number of examples of groups of finite $C$-width, and, in particular, we prove that the commutator subgroup $F^{\prime}$ of Thompson's group $F$ is a group of finite $C$-width. We also study the behaviour of the class of all groups of finite $C$-width under some group-theoretic constructions; it is established, for instance, that this class is closed under formation of group extensions.
\end{abstract}

\section{INTRODUCTION}

Let $G$ be a group and let $S$ be a generating set of $G$. Then the length $|g|_{S}$ of an element $g \in G$ with regard to $S$ is the least number of elements of $S \cup S^{-1}$ whose product is $g$ and the width $\operatorname{wid}(G, S)$ of $G$ with regard to $S$ is

$$
\operatorname{wid}(G, S)=\sup \left\{|g|_{S}: g \in G\right\} .
$$

Thus $\operatorname{wid}(G, S)$ is either the least natural number $k$ such that every element of $G$ is written as a product of at most $k$ elements of $S \cup S^{-1}$, or $\operatorname{wid}(G, S)=\infty$ if such $k$ does not exist. If the width $G$ with regard to $S$ is a finite number $k$, it is convenient to say that $G$ is generated by $S$ in $k$ steps. If, furthermore, $S=S^{-1}$ is a symmetric generating set, then $G=S^{k}$.

Recall that a group $G$ is said to be a group of finite width (or to satisfy the Bergman property), if $G$ has finite width with respect to every generating set. The first example of an infinite group of finite width has been given in 1980 by S. Shelah [16. In 2003 Bergman (see [5]) proved that all infinite symmetric groups are groups of finite width. Some questions Bergman included in his 2003 preprint of [5] initiated the search of new examples of groups of finite width. In particular, it has been discovered that the following groups are groups of finite width: the automorphism groups of 2-transitive linearly ordered sets [9], $\omega_{1}$-existentially closed groups [8], the autohomeomorphism groups of some topological spaces [10], the automorphism groups of infinite-dimensional vector spaces over arbitrary skew

2010 Mathematics Subject Classification. 20F05 (20F65). 
fields [17], the automorphism groups of infinitely generated free nilpotent groups [18], the automorphism groups of many $\omega$-stable and $\omega$-categorical structures [11] and so on.

The class of all groups of finite width has a number of attractive properties being, for instance, closed under homomorphic images and under formation of group extensions [5].

Naturally, one can weaken the condition requiring that a given group $G$ have finite width with regard to all generating sets (which leads to the notion of a group of finite width) by requiring that $G$ have finite width with regard to all generating sets satisfying a certain condition $\chi$, thereby getting the notion of a group of finite $\chi$-width.

In the present paper we study one of the natural choices for $\chi$ above, the condition $C=$ 'to be conjugation-invariant' (to be invariant under all inner automorphisms). Thus we say that a given group $G$ is a group of finite $C$-width if $G$ has finite width with regard to every conjugation-invariant generating set. Every group of finite width is of course a group of finite $C$-width, but the converse is not true (since, for instance, there are finitely generated infinite groups of finite $C$-width). The notion of a group of finite width and the notion of a group of finite $C$-width coincide on the class of all abelian groups; Bergman [5] proved that the abelian groups of finite width are exactly the finite ones.

It is easy to see that the special linear group $\operatorname{SL}_{n}(K)$ where $n \geqslant 3$ and $K$ is an arbitrary field is a group of finite $C$-width. Indeed, the conjugacy class $T \subseteq \mathrm{SL}_{n}(K)$ which consists of all transvections generates the group $G$ in $n$ steps: $\mathrm{SL}_{n}(F)=T^{n}$. On the other hand, if $S=S^{-1}$ is a symmetric, conjugationinvariant generating set of $\mathrm{SL}_{n}(K)$, then an appropriate power $S^{k}$ contains a transvection (and hence all transvections), whence $\operatorname{SL}_{n}(K)=\left(S^{k}\right)^{n}=T^{n}=S^{k n}$.

In the next section we shall give some other examples of groups of finite $C$ width. In particular, we shall prove that the commutator subgroup $F^{\prime}$ of Thompson's group $F$ is a group of finite $C$-width. The proof uses the ideas developed in the paper [6] by Burago, Ivanov and Polterovich. We shall also show that some groups of 'bounded' automorphisms of infinitely generated relatively free algebras are groups of finite $C$-width.

In the final section we analyze the behavior of the class of all groups of finite $C$-width under some group-theoretic constructions. It shall be proved that the class of all groups of finite $C$-width is closed, like the class of all groups of finite width, under formation of group extensions. We shall consider the question when a free product of nonidentity groups is a group of finite $C$-width. It turns out that the only, up to an isomorphism, free product of nonidentity groups which is a group of finite $C$-width is the group $\mathbf{Z}_{2} * \mathbf{Z}_{2}$. We also give a number of necessary and sufficient conditions for all functions $L: G \rightarrow \mathbf{R}$ on a given group $G$ such 
that

$$
\begin{aligned}
& L^{-1}(0)=\{1\}, \\
& L\left(g^{-1}\right)=L(g) \quad(g \in G), \\
& L(g h) \leqslant L(g)+L(h) \quad(g, h \in G), \\
& L\left(h g h^{-1}\right)=L(g) \quad(g, h \in G),
\end{aligned}
$$

called norms in [6], to be bounded from above. For instance, all norms on a given group $G$ are bounded from above if and only if $G$ is a group of finite $C$-width and every exhaustive chain $\left(N_{k}\right)$ of normal subgroups of $G$ terminates after finitely many steps. One more such a condition states that for every action of $G$ by isometries on a metric space $\langle M ; d\rangle$ such that

$$
d\left(g h g^{-1} a, a\right)=d(h a, a) \quad(a \in M ; g, h \in G),
$$

the diameters of all $G$-orbits are bounded.

\section{Examples of GRoups of Finite $C$-Width}

We shall say that a generating set $S$ of a given group $G$ is conjugation-invariant, if $S$ is invariant under all conjugations (inner automorphisms of $G$ ). A group $G$ is said to be a group of finite $C$-width if $G$ has finite width relative to every conjugation-invariant generating set.

The following simple sufficient condition for finiteness of $C$-width works, as we shall see, in many cases.

Lemma 2.1. Let a group $G$ be generated by finitely many conjugacy classes $C_{1}, \ldots, C_{m}$ in finitely many steps:

$$
\operatorname{wid}\left(G, C_{1} \cup \ldots \cup C_{m}\right)=N<\infty .
$$

Then $G$ is a group of finite $C$-width.

Proof. Suppose that $C_{k}=a_{k}^{G}$ is the conjugacy class of a certain element $a_{k} \in G$ $(k=1, \ldots, m)$. Consider a symmetric, conjugation-invariant generating set $S$ of $G$. For every $k=1, \ldots, m$, a suitable power $S^{p_{k}}$ of $S$ contains the element $a_{k}$. Set

$$
p=\max \left(p_{1}, \ldots, p_{m}\right) .
$$

Then

$$
C_{1} \cup \ldots \cup C_{m} \subseteq S^{p}
$$

and it follows that $G=\left(S^{p}\right)^{N}=S^{p N}$.

Now let us consider examples of groups of finite $C$-width. 
1) We have already mentioned in the introduction that the special linear group $\mathrm{SL}_{n}(K)$ over a field $K$ of dimension $n \geqslant 3$ is a group of finite $C$-width, since this group is generated by the conjugacy class of all transvections in $n$ steps.

2) Lemma 2.1 and Example 1.1 from [6] imply that the special linear group $\mathrm{SL}_{n}(\mathbf{Z})$ where $n \geqslant 3$ over the ring of integers $\mathbf{Z}$ is also a group of finite $C$-width. In fact this result has been proven in [6] (though in different terms). Indeed, it is known that the group $\mathrm{SL}_{n}(\mathbf{Z})$ is generated by the family of all elementary transvections in some $K_{n}<\infty$ steps (see [14]). An elementary transvection $t_{i j}(m)$, where $i, j$ with $1 \leqslant i, j \leqslant n$ are distinct indices and $m \in \mathbf{Z}$, is the matrix

$$
t_{i j}(m)=I+m I_{i j}
$$

Observe that the group $\mathrm{SL}_{n}(\mathbf{Z})$ is also generated by the conjugacy class $T$ of the transvection $t_{12}(1)$. On the other hand, the following well-known formula

$$
t_{i j}(m)=\left[t_{i k}(1), t_{k j}(m)\right] \quad(m \in \mathbf{Z}),
$$

where $1 \leqslant i, j, k \leqslant n$ are pairwise distinct indices, implies that every elementary transvection is a product of two elements of $T$. Thus the group $\mathrm{SL}_{n}(\mathbf{Z})$ is generated by the conjugacy class $T$ in at most $2 K_{n}$ steps.

3) Among known examples of groups of finite width, quite a few are groups that are generated by a single conjugacy class. For instance, the mentioned property is shared by the infinite symmetric groups, the automorphism groups of infinite dimensional vector spaces over skew fields, the automorphism groups of infinitely generated free nilpotent groups and so on.

4) Every nonidentity algebraically closed group $G$ is generated by the conjugacy class of any nontrivial element in two steps [12, Cor. 2]. Accordingly, $G$ is a group of finite $C$-width.

5) Formally, if a given group has only finitely many conjugacy classes, then this group is a group of finite $C$-width.

6) Evidently, any homomorphic image (any quotient group) of a group of finite $C$-width is also a group of finite $C$-width.

Let $G$ be a group. The commutator subgroup of a subgroup $H$ of $G$ will be denoted by $H^{\prime}$. Our next example is

2.1. The commutator subgroup $F^{\prime}$ of Thompson's group $F$. By the definition, subgroups $H_{1}, H_{2}$ of a group $G$ are said to be commuting if $\left[H_{1}, H_{2}\right]=\{1\}$, that is, if every element of $H_{1}$ commutes with every element of $H_{2}$.

For convenience's sake, we reproduce some definitions and results from [6] we are going to use below. 
Let $H \leqslant G$, let $f$ be an element of $G$, and let $m \geqslant 1$ be a natural number. Then $f$-displaces $H$, if the subgroups

$$
H, f H f^{-1}, f^{2} H f^{-2}, \ldots, f^{m} H f^{-m}
$$

are pairwise commuting [6. Sect. 2.1]. We shall say that $f \omega$-displaces $H$ if $f$ $m$-displaces $H$ for all $m \geqslant 1$.

An element $x \in G$ is said to be an $f$-commutator if $x$ is conjugate to a commutator of the form $[f, g]$ where $g \in G$. Observe that the inverse of an $f$-commutator is also an $f$-commutator.

Proposition 2.2. (i). Suppose that $f m$-displaces a subgroup $H \leqslant G$ for some $m \geqslant 2$. Then every element of $H^{\prime}$ which has commutator length $m$ in $H$ can be written as a product of an $f$-commutator and a commutator of some elements of $G$ (see the proof of Theorem 2.2 (i) in [6]).

(ii). Suppose that $f \in G \omega$-displaces a subgroup $H$ of $G$. The commutator length (in $G$ ) of every element $h \in H^{\prime}$ is at most two [6. Lemma 2.2].

(iii). Suppose that $f m$-displaces a subgroup $H$ of $G$ where $m \geqslant 2$. Then every commutator of elements of $H$ is a product of two $f$-commutators [6, Lemma 2.7].

Proposition 2.3. Let $f$ be an arbitrary nonidentity element of the commutator subgroup $F^{\prime}$ of Thompson's group $F$. Then every element of $F^{\prime}$ is a product of at most six elements of $C(f)^{ \pm 1}$ where $C(f)$ is the conjugacy class of $f$ in $F^{\prime}$. Consequently, the commutator subgroup $F^{\prime}$ of Thompson's group $F$ is a group of finite $C$-width.

Proof. We shall work with the group $\mathrm{PL}_{2}([0,1])$, one of the standard realizations of $F$ [4, 17]. So we assume that $F=\mathrm{PL}_{2}([0,1])$.

The next result is well-known (folklore; see Proposition 8.1 in [1]).

Lemma 2.4. Every commutator $[a, b] \in F^{\prime}$ where $a, b \in F$ can be written in the form $\left[a_{1}, b_{1}\right]$ where $a_{1}, b_{1}$ are already elements of $F^{\prime}$.

Recall that every element of $F^{\prime}$ acts identically on a certain closed segment $[0, \beta]$ and on a certain closed segment $[\gamma, 1]$ where $0<\beta<\gamma<1$ (see, e.g., [4, Cor. 1.3.4]). Let $f$ be a nonidentity element of $F^{\prime}$. Suppose that $0<\alpha_{0} \in[0,1]$ is the first nontrivial fixed point of $f$, that is, the fixed point of $f$ such that the interval $\left[0, \alpha_{0}\right]$ is fixed by $f$ pointwise, and every open neighbourhood of $\alpha_{0}$ has points that are not fixed by $f$. Let, further, $\alpha_{1}<1$ be the next fixed point of $f$, that is, the point of $(0,1)$ such that there are no fixed points of $f$ in the open interval $\left(\alpha_{0}, \alpha_{1}\right)$. Take a dyadic point $\alpha \in\left(\alpha_{0}, \alpha_{1}\right) \cap \mathbf{Z}\left[\frac{1}{2}\right]$. Then either

$$
\alpha_{0}<\alpha<f \alpha<f^{2} \alpha<\ldots<f^{n} \alpha<\ldots<\alpha_{1},
$$


in the case when $\alpha<f \alpha$, or

$$
\alpha_{0}<\ldots<f^{n} \alpha<\ldots<f^{2} \alpha<f \alpha<\alpha<\alpha_{1}
$$

in the case when $\alpha>f \alpha$, since $f$ is an increasing function.

Consider the open interval $J$ with endpoints $\alpha$ and $f \alpha$. Clearly, the intervals

$$
J, f J, f^{2} J, \ldots, f^{m} J, \ldots
$$

all containing in the open interval $\left(\alpha_{0}, \alpha_{1}\right)$, are pairwise disjoint.

Let $H$ be the subgroup of all members $F^{\prime}$ whose supports are in $J$. It is easy to see that $H$ is isomorphic to $F$, since

$$
\mathrm{PL}_{2}(\bar{J}) \cong \mathrm{PL}_{2}([0,1])=F,
$$

where $\bar{J}$ is the closure of $J$ [4, Prop. 1.4.4].

Recall that for every $n \geqslant 1$, the group $F^{\prime}$ acts transitively on the family of $n$-element ordered tuples of $(0,1) \cap \mathbf{Z}[1 / 2]$ [7, Lemma 4.2]. It follows that every finite tuple of elements of $F^{\prime}$ can be taken into $H^{\prime}$ by conjugation by a suitable element of $F^{\prime}$.

As open intervals in (2.1) are pairwise disjoint, we get that $f \omega$-displaces $H$ in $F^{\prime}$. Using Lemma 2.4 and part (i) of Proposition 2.2, we can write any element $h \in H^{\prime}$ as a product of an $f$-commutator and a commutator of elements of $F^{\prime}$ :

$$
h=\left[f, b_{1}\right]^{c_{1}}\left[b_{2}, b_{3}\right] .
$$

Conjugating then the elements $b_{2}, b_{3}$ by an appropriate $c \in F^{\prime}$, we obtain the commutator

$$
\left[b_{2}^{c}, b_{3}^{c}\right]
$$

of elements of $H$. By part (iii) of Proposition 2.2, the latter commutator is a product of two $f$-commutators. Then we deduce from $(2.2)$, that $h^{c}$ is a product of three $f$-commutators. Accordingly, $h$ is a product of three $f$-commutators, and then every element of $F^{\prime}$ is a product of three $f$-commutators. Clearly, a product of three $f$-commutators is a product of six elements of $C(f)^{ \pm 1}$, where $C(f)$ is the conjugacy class of $f$ in $F^{\prime}$.

Remarks 2.5. (i) As it has been demonstrated in the course of the proof of the proposition, every element of $F^{\prime}$ is a product of two commutators (folklore; see [1. Prop. 8.1]). The problem whether every element of $F^{\prime}$ is a commutator is open.

(ii) The group $F$ itself is not a group of finite $C$-width, for the abelianization $F / F^{\prime} \cong \mathbf{Z}^{2}$ of $F$ is infinite. As for $F^{\prime}$, it is not a group of finite width, since its width relative to the generating set

$$
\left\{x_{n} x_{n+1}^{-1}: n=0,1,2, \ldots\right\}
$$

is infinite. 
(iii). Proposition 2.3 implies the well-known fact of simplicity of the group $F^{\prime}$.

2.2. Subgroups of 'bounded' automorphisms of relatively free algebras. Let $\mathfrak{V}$ be a variety of algebras and let $\mathcal{F} \in \mathfrak{V}$ be a free $\mathfrak{V}$-algebra of infinite rank $\varkappa$. Consider a basis $\mathcal{B}$ of $\mathcal{F}$ and an infinite cardinal $l<\varkappa$. For every $\varphi \in \operatorname{Aut}(\mathcal{F})$, define the support of $\varphi$ as

$$
\operatorname{supp}(\varphi)=\{b \in \mathcal{B}: \varphi b \neq b\} .
$$

Set

$$
\operatorname{Aut}_{l, \mathcal{B}}(\mathcal{F})=\{\varphi \in \operatorname{Aut}(\mathcal{F}):|\operatorname{supp}(\varphi)| \leqslant l\} .
$$

Clearly, $G=\operatorname{Aut}_{l, \mathcal{B}}(\mathcal{F})$ is a subgroup of the automorphism group $\operatorname{Aut}(\mathcal{F})$ of $\mathcal{F}$, and every element of $G$ fixes the 'most' of elements of $\mathcal{B}$.

Proposition 2.6. The group $G=\operatorname{Aut}_{l, \mathcal{B}}(\mathcal{F})$ is a group of finite $C$-width.

Proof. Recall that a subset $J$ of an infinite set $I$ is called a moiety of $I$ if $|J|=$ $|I \backslash J|$.

We term an automorphism of a given relatively free algebra permutational if it fixes setwise a certain basis of this algebra.

Let $\mathcal{M}$ be a relatively free algebra with an infinite basis $\mathcal{X}$. Let $\mathcal{Y}$ be a moiety of $\mathcal{X}$. Consider a permutational automorphism $\pi$ of $\mathcal{M}$ which acts on $\mathcal{Y}$ as an involution without fixed points and which fixes pointwise all elements of $\mathcal{X} \backslash \mathcal{Y}$. As it has been demonstrated in the proof of part (i) of Theorem 1.5 in [18], every automorphism $\mathcal{M}$ which fixes the subalgebra $\langle\mathcal{Y}\rangle$ setwise and takes every element of the set $\mathcal{X} \backslash \mathcal{Y}$ to itself is a product of at most four conjugates of $\pi$.

Choose a subset $\mathcal{C}$ of the basis $\mathcal{B}$ of cardinality $l$ and a moiety $\mathcal{C}_{0}$ of $\mathcal{C}$. Consider a permutational automorphism $\pi^{*} \in G$ which

- fixes the set $\mathcal{B} \backslash \mathcal{C}_{0}$ pointwise;

- acts on the set $\mathcal{C}_{0}$ as an involution without fixed points.

Let $H$ be a subgroup of $G$ consisting of all automorphisms of $\mathcal{F}$ preserving the subalgebra $\langle\mathcal{C}\rangle$ generated by $\mathcal{C}$ as a set and taking to themselves all elements of $\mathcal{B} \backslash \mathcal{C}$. Clearly, for every $\sigma \in G$ there is a permutational automorphism $\rho \in G$ such that the conjugate $\sigma_{0}$ of $\sigma$ by $\rho$ is in $H$, fixes the subalgebra $\left\langle\mathcal{C}_{0}\right\rangle$ setwise, and fixes the set $\mathcal{B} \backslash \mathcal{C}_{0}$ pointwise.

Then we obtain, as a corollary of the above-quoted result from [18], that $\sigma_{0}$ is a product of at most four conjugates of $\pi^{*}$ in $H \cong \operatorname{Aut}(\langle\mathcal{C}\rangle)$. Consequently, $\sigma$ is a product of at most four conjugates of $\pi^{*}$ in $G$, whence $G=C\left(\pi^{*}\right)^{4}$ where $C\left(\pi^{*}\right)$ is the conjugacy class of $\pi^{*}$ in $G$.

Thus the group $G$ is generated by the class $C\left(\pi^{*}\right)$ in at most of four steps, and then it is a group of finite $C$-width by Lemma 2.1 . 


\section{3. $C$-Width And Group-Theoretic COnstructions}

3.1. Group extensions. The proof of the fact that the class of all groups of finite width is closed under formation of group extensions given by Bergman in [5] is based upon the following statement.

Lemma 3.1. [5, Lemma 7] Let $U=U^{-1}$ be a symmetric generating set and let $H$ be a subgroup of given group $G$ such that for some natural number $n$

$$
H g \cap U^{n} \neq \varnothing
$$

for all $g \in G$. Then $H$ is generated by those elements $G$ that have length $\leqslant 2 n+1$ relative to $U$ :

$$
H=\left\langle H \cap U^{2 n+1}\right\rangle .
$$

Proposition 3.2. Let $G$ be a group and let $H$ be a normal subgroup of $G$ such that both the subgroup $H$ and the quotient group $G / H$ are groups of finite $C$-width. Then $G$ is also a group of finite $C$-width.

Proof. Let $U$ be a symmetric, conjugation-invariant generating set of $G$ and let $f: G \rightarrow G / H$ be the natural homomorphism from $G$ onto $G / H$. Clearly, $f(U)$ is also a conjugation-invariant generating set of the group $f(G)=G / H$. But then

$$
\operatorname{wid}(f(G), f(U))=n<\infty
$$

and we are in the conditions of Lemma 3.1. Evidently, $H \cap U^{2 n+1}$ is a conjugationinvariant generating set of $H$ :

$$
\left(H \cap U^{2 n+1}\right)^{h}=H^{h} \cap\left(U^{2 n+1}\right)^{h}=H \cap U^{2 n+1} \quad(h \in H) .
$$

Hence, by the conditions,

$$
\operatorname{wid}\left(H, H \cap U^{2 n+1}\right)=m<\infty,
$$

whence

$$
\operatorname{wid}(G, U) \leqslant \operatorname{wid}(f(G), f(U))+\operatorname{wid}(H, U) \leqslant n+(2 n+1) m<\infty .
$$

We then obtain as a corollary that the class of all groups of finite $C$-width is closed under formation of group extensions, and, in particular, it is closed under formation of direct products and under formation of group extensions by finite groups. 
3.2. Free products. Essentially, the following result states that the only free product of nonidentity groups which is a group of finite $C$-width is, up to an isomorphism, the free product $\mathbf{Z}_{2} * \mathbf{Z}_{2}$.

Proposition 3.3. The free product $\prod_{i \in I}^{*} G_{i}$ of a family $\left\{G_{i}: i \in I\right\}$ of nonidentity groups is a group of finite $C$-width if and only if $|I|=2$ and both groups participating in the free product are of order two.

Proof. $(\Leftarrow)$. Consider groups $A=\langle a\rangle$ and $B=\langle b\rangle$, both isomorphic to the group $\mathbf{Z}_{2}$. Let $w \in A * B$ be a reduced word in letters $a, b$ which begins with $a$. Then

$$
w=(a b)^{k} a, \text { or } w=(a b)^{k}
$$

for a suitable natural number $k$. It is easy to see that each word of the form $(a b)^{k} a$ is conjugate either to $a$, or to $b$. Let $k \geqslant 1$. Then

$$
(a b)^{k}=(a b)^{k-1} a b=(a b)^{k-1} a \cdot b
$$

and the element $(a b)^{k}$ is a product of at most two conjugates of elements of $\{a, b\}$. The argument in the case when $w$ begins with $b$ is similar. Therefore the group $A * B$ is generated in two steps by the union of the conjugacy class of $a$ and the conjugacy class of $b$. Apply Lemma 2.1 to complete the proof.

$(\Rightarrow)$. Recall that a map $\Delta: H \rightarrow \mathbf{Z}$ from a given group $H$ into $\mathbf{Z}$ is called a quasi-homomorphism if there is a constant $C$ such that

$$
\Delta(a b) \leqslant \Delta(a)+\Delta(b)+C \quad(a, b \in H) .
$$

Fix a family $\left\{G_{i}: i \in I\right\}$ of nonidentity groups, and let $G$ denote the free product $\prod_{i \in I}^{*} G_{i}$. An element $p$ of the free product $G$ is called a palindrome if the reduced word representing $p$ (whose syllables are nonidentity elements of factors $G_{i}$ ) is read the same way forwards and backwards. Thus if $p \in G$ and

$$
p=v_{1} \ldots v_{n}
$$

where $v_{k}$ are nonidentity elements of free factors $G_{i}$ such that the elements $v_{m}, v_{m+1}$ lie in distinct free factors for all $m$, then $p$ is a palindrome if and only if

$$
v_{1} \ldots v_{n}=v_{n} \ldots v_{1} \text {. }
$$

In [3] quasi-homomorphisms have been used to show that all free products of nonidentity groups that are not isomorphic to the group $\mathbf{Z}_{2} * \mathbf{Z}_{2}$ have infinite width relative to the (generating) set of all palindromes, or, in other words, infinite palindromic width.

It particular, it is proved in [3] that if the free product $G=\prod_{i \in I}^{*} G_{i}$ where $|I| \geqslant 2$ is such that

$$
\text { at least one free factors } G_{i} \text { has an element of order } \geqslant 3
$$

then there is a quasi-homomorphism $\Delta_{1}: G \rightarrow \mathbf{Z}$ with the following properties: 
(a) $\Delta_{1}(a b) \leqslant \Delta_{1}(a)+\Delta_{1}(b)+9$ for all $a, b \in G$;

(b) the value $\Delta_{1}$ at any palindrome of $G$ is at most 2;

(c) $\Delta_{1}$ is not bounded from above.

(see [3, pp. 203-204]).

1) Suppose then that our free product $G$ has the property (3.1). The reader can verify quite easily that it follows from the definition of $\Delta_{1}$ in [3] that

$$
\Delta_{1}\left(a b a^{-1}\right) \leqslant \Delta_{1}(b)+9 \quad(a, b \in G) .
$$

By (b), this implies that the value of $\Delta_{1}$ at any conjugate of a palindrome in $G$ is at most 11 . Now, were the width of $G$ relative to the family of all conjugates of palindromes finite, the quasi-homomorphism $\Delta_{1}$ would be bounded on $G$, contradicting (c). Therefore every free product of nonidentity groups with (3.1) is not a group of finite $C$-width.

2) Now let $G \approx \mathbf{Z}_{2} * \mathbf{Z}_{2}$ not satisfy (3.1). Then $G$ is a free product of nontrivial abelian groups of exponent two. It is easy to see that in any free product of abelian groups of exponent two, a conjugate of a palindrome is a palindrome, too. Thus the set of all palindromes of $G$ is invariant under all conjugations. As we have mentioned above, the palindromic width of $G$ is infinite, and hence $G$ is not a group of finite $C$-width.

3.3. Functions similar to length functions and cofinalities. Basing on the ideas from the paper [5] by Bergman, several authors obtained a number of necessary and sufficient conditions for all functions $L: G \rightarrow \mathbf{R}$ on a given group $G$ such that

$$
\begin{array}{ll}
L^{-1}(0)=\{1\}, & \\
L\left(g^{-1}\right)=L(g) & \\
L(g h) \leqslant L(g)+L(h) & (g, h \in G),
\end{array}
$$

to be bounded from above. For instance, this takes place if and only if $G$ is a group of finite width and the cofinality $\mathrm{cf}(G)$ is uncountable [9, 10]; another such criterion states that every action of $G$ by isometries on a metric space has bounded orbits [8].

By the definition, the cofinality $\operatorname{cf}(G)$ of an infinitely generated group $G$ is the least cardinal $\lambda$ such that $G$ can be written as the union of a chain of cardinality $\lambda$ of its proper subgroups (observe that no finitely generated group can be written as the union of a chain of proper subgroups). In the case when $\operatorname{cf}(G)>\aleph_{0}$, a group $G$ is said to be a group of uncountable cofinality. For instance, the symmetric group $\operatorname{Sym}(X)$ of an infinite set $X$ is a group of uncountable cofinality, since $\operatorname{cf}(\operatorname{Sym}(X))>|X|[13,5]$. 
In accordance with the terminology introduced in [10], if $G$ is a group of finite width and of uncountable cofinality, $G$ is called a group of strong uncountable cofinality.

As we mentioned in the introduction, the paper [6] contains a number of results on functions (termed norms in [6]) $L: G \rightarrow \mathbf{R}$ on groups satisfying the conditions (3.2) and taking constant values on conjugacy classes, that is, satisfying the additional condition

$$
L\left(g h g^{-1}\right)=L(h) \quad(g, h \in G) .
$$

We shall provide below some necessary and sufficient conditions for all norms on a given group $G$ to be bounded from above. Our conditions are in fact naturallyweakened versions of conditions, equivalent to strong uncountable cofinality, that can be found in papers $[8,10,15]$.

Proposition 3.4. Let $G$ be a group. Then the following are equivalent:

(i). $G$ is a group of finite $C$-width and every exhaustive chain $\left(N_{k}\right)$

$$
N_{0} \leqslant N_{1} \leqslant \ldots \leqslant N_{k} \leqslant \ldots \leqslant G
$$

of normal subgroups of $G$ (every increasing chain of normal subgroups whose union is $G$ ) terminates after finitely many steps;

(ii). Every exhaustive chain $\left(U_{k}\right)$

$$
U_{0} \subseteq U_{1} \subseteq \ldots \subseteq U_{k} \subseteq \ldots \subseteq G
$$

of subsets of $G$ such that for every $i \in \mathbf{N}$

- $U_{i}$ closed under taking inverses;

- $U_{i}$ is conjugation-invariant;

- the product $U_{i} U_{i}$ is contained in a suitable $U_{k}$

terminates after finitely many steps;

(iii). Orbits of every action of $G$ by isometries on a metric space $\langle M, d\rangle$ such that

$$
d\left(a, g h g^{-1} a\right)=d(a, h a) \quad(a \in M ; g, h \in G)
$$

have bounded diameters;

(iv). Every function $L: G \rightarrow \mathbf{R}$ taking constant values on conjugacy classes of $G$ and such that

- $L(g)=0$ if and only if $g=1$;

- $L(g h) \leqslant L(g)+L(h)$ for all $g, h \in G$

is bounded from above. 
Proof. (i) $\Rightarrow$ (ii). Clearly, the chain of subgroups of $G$ generated by sets $U_{i}$,

$$
\left\langle U_{0}\right\rangle \leqslant\left\langle U_{1}\right\rangle \leqslant \ldots \leqslant\left\langle U_{k}\right\rangle \leqslant \ldots \leqslant G
$$

is an exhaustive chain of normal subgroups of $G$. Then $G=\left\langle U_{j}\right\rangle$ for a suitable natural number $j$, and hence $U_{j}$ is a symmetric, conjugation-invariant generating set of $G$. As $G$ is a group of finite $C$-width, $G=U_{j}^{s}$. By the conditions on the chain $\left(U_{k}\right)$, the power $U_{j}^{s}$ is contained in some $U_{m}$ for an appropriate $m \in \mathbf{N}$, whence $U_{m}=G$. Set

(ii) $\Rightarrow$ (iii). Let $a$ be an arbitrary element of a metric space $M$ satisfying (iii).

$$
U_{n}=\{g \in G: d(a, g a) \leqslant n\} \quad(n \in \mathbf{N}) .
$$

It follows from (iii) that every $U_{n}$ is conjugation-invariant. Let $g, h \in U_{n}$. Then we have that

$$
d(a, g h a) \leqslant d(a, g a)+d(g a, g h a)=d(a, g a)+d(a, h a) \leqslant n+n=2 n .
$$

Consequently, $U_{n} U_{n} \subseteq U_{2 n}$. As the chain $\left(U_{n}\right)$ terminates, we get that $G=U_{m}$ for some $m \in \mathbf{N}$. Hence

$$
d(a, g a) \leqslant m
$$

for all $g \in G$. Thus the diameter of the orbit $\{g a: g \in G\}$ of $a \in M$ is at most $2 m$.

$$
\text { (iii) } \Rightarrow \text { (iv). Let } a, b \in G \text {. Set }
$$

$$
d(a, b)=L\left(a b^{-1}\right) .
$$

It is easy to see that $d$ is a metric on $G$ satisfying the conditions in (iii) for the left action $G$ on itself. Indeed, we have that

$$
\begin{aligned}
& d(a, b)=0 \Longleftrightarrow L\left(a b^{-1}\right)=0 \Longleftrightarrow a b^{-1}=1 \Longleftrightarrow a=b, \\
& d(a, b)=L\left(a b^{-1}\right)=L\left(b a^{-1}\right)=d(b, a), \\
& d(a, b)=L\left(a b^{-1}\right)=L\left(a c^{-1} \cdot c b^{-1}\right) \leqslant L\left(a c^{-1}\right)+L\left(c b^{-1}\right)=d(a, c)+d(c, b), \\
& d(g a, g b)=L\left(g a b^{-1} g^{-1}\right)=L\left(a b^{-1}\right)=d(a, b), \\
& d\left(a, g h g^{-1} a\right)=L\left(g h g^{-1}\right)=L(h)=d(a, h a)
\end{aligned}
$$

for all $a, b, g, h \in G$. Then the orbit of $1 \in G$ under the left action of $G$ on itself has a bounded diameter $m \in \mathbf{N}$, or

$$
L(g)=d(g 1,1) \leqslant m \quad(g \in G) .
$$

(iv) $\Rightarrow$ (i). Let $S=S^{-1}$ be a symmetric, conjugation-invariant generating set of $G$. Then the function

$$
L_{1}(g)=|g|_{S} \quad(g \in G)
$$


that is, the length function with regard to $S$, which meets all conditions mentioned in (iv), must be bounded from above by some natural number $m$. Accordingly, $G=S^{m}$.

Let further $\left(N_{k}\right)$ be an exhaustive chain of normal subgroups of $G$. For every $g \in G$ set

$$
L_{2}(g)=\min \left\{k \in \mathbf{N}: g \in N_{k}\right\} \quad(g \in G) .
$$

It is readily seen that $L_{2}$ satisfies all conditions in (iv). For example,

$$
L_{2}(a b) \leqslant \max \left(L_{2}(a), L_{2}(b)\right) \leqslant L_{2}(a)+L_{2}(b)
$$

for all $a, b \in G$. One again concludes that $L_{2}$ is bounded from above by a certain natural number $m$, whence $G=N_{m}$.

Remark 3.5. In the case when $G$ is a simple group, Proposition 3.4 provides a criterion of finiteness of $C$-width of $G$.

In the conclusion of the section we shall discuss some notion which generalizes both the notion of a group of finite width and the notion of a group of finite $C$ width. Let $G$ be a group and let $\Sigma \leqslant \operatorname{Aut}(G)$ be a subgroup of the automorphism group of $G$. We say that $G$ has finite $\Sigma$-width if $G$ has finite width with respect to all $\Sigma$-invariant generating sets. Clearly, the case when $\Sigma=\{$ id $\}$ corresponds to the notion of a group of finite width, and the case when $\Sigma=\operatorname{Inn}(G)$ to that one of finite $C$-width.

Intuitively, the greater the (setwise) stabilizer in $\operatorname{Aut}(G)$ of a given set $S$ of generators of $G$, the more 'massive' $S$ appears to be with the 'point of view' of the automorphism group of $G$. Thus if $G$ has finite $\Sigma$-width in the case when $\Sigma=\operatorname{Aut}(G)$, it has finite width with regard to all 'most massive' generating sets.

One can, as we did in Proposition 3.4, add to the condition of finiteness of $\Sigma$-width the condition of termination of all exhaustive chains of $\Sigma$-invariant subgroups of $G$. Modifying then the formulation of Proposition 3.4 accordingly, one can obtain necessary and sufficient conditions for $G$ to have finite $\Sigma$-width and, simultaneously, to satisfy the condition of termination of all exhaustive chains of $\Sigma$-invariant subgroups. For instance, the analogue of the part (iii) of Proposition 3.4 is as follows: every action of $G$ by isometries on a metric space $\langle M, d\rangle$ for which

$$
d(\sigma(g) a, a)=d(g a, a), \quad(a \in M, \sigma \in \Sigma, g \in G)
$$

has bounded orbits.

The case when $\Sigma$ is equal to the full automorphism group $\operatorname{Aut}(G)$ of $G$ seems to be quite interesting. Simplifying the terminology somewhat, we say that $G$ has finite Aut-width if $G$ has finite width with respect to every generating set which is invariant under all automorphisms of $G$. 
Our final result shows that the class of all groups of finite Aut-width does not have some attractive properties that its counterparts, the classes of all groups of finite width and all groups of finite of $C$-width, have (in particular, this class is not closed under homomorphic images). Nevertheless, the property of having/not having finite Aut-width can be used to distinguish between the isomorphism types of groups.

Proposition 3.6. A free group $F$ is a group of finite Aut-width if and only if its rank is infinite.

Proof. Suppose that $F$ is of finite rank. Consider the (generating) set $P$ of all primitive elements of $F$. Clearly, $P$ is invariant under all automorphisms of $F$, but the width of $F$ relative to $P$ is infinite [2, Th. 2.1].

Now let $F$ be of infinite rank. This time, the width of $F$ with regard to the set of all primitive elements is two [2, Th. 2.1]. Consider a symmetric generating set $S$

of $F$ which is invariant under all automorphisms of $F$. Then a certain power $S^{k}$ of $S$ contains a primitive element $p \in F$. As $S$ is invariant under automorphisms of $F$, the said power of $S$ contains all primitive elements. Consequently, $F=S^{2 k}$.

\section{REFERENCES}

[1] T. Altinel, A. Muranov, Interpretation de l'arithmetique dans certains groupes de permutations affines par morceaux d'un intervalle. J. Inst. Math. Jussieu 8 (4) (2009) 623-652.

[2] V. Bardakov, V. Shpilrain, V. Tolstykh, On the palindromic and primitive widths of a free group, J. Algebra 285 (2) (2005) 574-585.

[3] V. Bardakov, V. Tolstykh, The palindromic width of a free product of groups, J. Aust. Math. Soc. 81 (2) (2006) 199-208.

[4] J. Belk, Thompson's group F, Ph. D. Thesis, Cornell Univ., 2004, available at http://www.math.cornell.edu/ belk/Thesis.pdf.

[5] G. Bergman, Generating infinite symmetric groups, Bull. London Math. Soc. 38 (3) (2006) 429-440.

[6] D. Burago, S. Ivanov, L. Polterovich, Conjugation-invariant norms on groups of geometric origin, in Groups of diffeomorphisms, Adv. Stud. Pure Math., 52, Math. Soc. Japan, Tokyo, 2008, 221-250.

[7] J. Cannon, W. Floyd, W. Parry, Introductory notes to Richard Thompson's groups, Enseign. Math. 42 (1996) 215-256.

[8] Y. de Cornulier, Strongly bounded groups and infinite powers of finite groups, Comm. Algebra 34 (7) (2006), 2337-2345.

[9] M. Droste, W. C. Holland, Generating automorphism groups of chains, Forum Math 17 (2005) 699-710.

[10] M. Droste, R. Göbel, Uncountable confinalities of permutations groups, J. London Math. Soc. 71 (2) 335-344.

[11] A. Kechris, C. Rosendal, Turbulence, amalgamation, and generic automorphisms of homogeneous structures, Proc. Lond. Math. Soc. 94 (2007) 302-350.

[12] A. Macintyre, On algebraically closed groups, Ann. of Math., 96 (1972) 53-97.

[13] H. D. Macpherson, P. M. Neumann, Subgroups of infinite symmetric groups, J. London Math. Soc. 42 (1990) 64-84. 
[14] D. W. Morris, Bounded generation of $\operatorname{SL}(n, A)$ (after D. Carter, G. Keller, and E. Paige), New York J. Math. 13 (2007) 383-421.

[15] C. Rosendal, A topological version of the Bergman property, Forum Math. 21 (2) (2009) 299-332.

[16] S. Shelah, On a problem of Kurosh, Jónsson groups, and applications, in Stud. Logic Foundations Math. 95, North-Holland, Amsterdam-New York, 1980, 373-394.

[17] V. A. Tolstykh, Infinite-dimensional general linear groups are groups of finite width, Sib. J. Math. 47 (5) (2006) 1160-1166.

[18] V. Tolstykh, On the Bergman property for the automorphism groups of relatively free groups, J. London Math. Soc. 73 (3) (2006) 669-680.

Valery Bardakov, Sobolev Institute of Mathematics, Siberian Branch of the Russian Academy of Sciences, 630090 Novosibirsk, Russia

E-mail address: bardakov@math.nsc.ru

Vladimir Vershinin, Department of Mathematics, University Montpellier II, Place Eugène Bataillon, 34095 Montpellier Cedex 5, France

E-mail address: vershini@math.univ-montp2.fr

Vladimir Tolstykh, Department of Mathematics, Yeditepe University, 34755 KAYIŞDAĞI, ISTANBUL, TURKEY

E-mail address: vtolstykh@yeditepe.edu.tr 